\title{
Resonant harmonic response in tapping-mode atomic force microscopy
}

\author{
Ozgur Sahin,* Calvin F. Quate, and Olav Solgaard \\ E. L. Ginzton Laboratory, Stanford University, Stanford, California 94305, USA
}

Abdullah Atalar

Electrical and Electronics Engineering Department, Bilkent University, Bilkent, 06533 Ankara, Turkey

(Received 4 December 2003; published 28 April 2004)

\begin{abstract}
Higher harmonics in tapping-mode atomic force microscopy offers the potential for imaging and sensing material properties at the nanoscale. The signal level at a given harmonic of the fundamental mode can be enhanced if the cantilever is designed in such a way that the frequency of one of the higher harmonics of the fundamental mode (designated as the resonant harmonic) matches the resonant frequency of a higher-order flexural mode. Here we present an analytical approach that relates the amplitude and phase of the cantilever vibration at the frequency of the resonant harmonic to the elastic modulus of the sample. The resonant harmonic response is optimized for different samples with a proper design of the cantilever. It is found that resonant harmonics are sensitive to the stiffness of the material under investigation.
\end{abstract}

DOI: 10.1103/PhysRevB.69.165416

PACS number(s): 68.37.Ps, 07.79.Lh, 87.64.Dz

\section{INTRODUCTION}

The atomic force microscope ${ }^{1}$ (AFM) is primarily a tool for characterizing surface topography, but there is always a strong interest in using this technique to study the mechanical properties of samples at the nanoscale. A method for probing elastic and viscoelastic surface properties will enhance our ability to characterize materials, map variations in chemical composition, and investigate the properties of nanostructures. The techniques for measuring these properties include force modulation microscopy, ${ }^{2}$ the force curve method, ${ }^{3}$ nanoindentation, ${ }^{4}$ pulsed force mode, ${ }^{5}$ and ultrasonic force microscopy. ${ }^{6-8}$ These techniques measure the elastic properties either directly by indenting the surface with a force applied to the tip or indirectly by monitoring the response of the cantilever with the tip in contact with the surface. The latter is more sensitive to the local stiffness of the samples, ${ }^{9-11}$ but sometimes results in damage. Probing the surface with the tapping mode for the AFM (Ref. 12) is a more gentle procedure that largely eliminates damage to the sample.

In tapping mode the cantilever is driven at the resonant frequency of the fundamental mode with the tip periodically tapping on the surface. A feedback loop is used to maintain the excursion of the oscillating tip at a constant level. The variations in both amplitude and phase of the feedback signal reveal the surface topography. Images obtained with the phase signal exhibit good contrast for different materials. ${ }^{13,14}$ Unfortunately, multiple sources of dissipation, such as capillary forces, ${ }^{15}$ viscoelasticity of samples, and electronic dissipation, make it difficult to interpret the phase signal and relate it to material properties. Balantekin and Atalar ${ }^{16}$ have suggested that the elastic and viscoelastic properties can be inferred from the amplitude and phase of the cantilever motion if the mechanical parameters of the cantilevers are known. Their model is limited to hydrophobic surfaces since it does not account for the capillary forces between the tip and the sample. Stark et al. have shown that the phase signal is influenced by the topographical variations, ${ }^{17}$ which makes it difficult to interpret the image. Recently, Rodriguez and Garcia ${ }^{18}$ proposed excitation of the first modes of the cantilever to create coupled anharmonic oscillators with high sensitivity to variations in the attractive component of the tipsample forces that depend on the chemical composition of the sample.

There is a wealth of information in the harmonics generated when the tip periodically taps on the sample surface. ${ }^{19-21}$ Heretofore, this information has been hidden beneath the noise floor because of the rapid decay of the amplitude of the harmonics. ${ }^{22}$ Here we show that a simple modification of the cantilever enhances the amplitude of a selected harmonic and increases the signal-to-noise ratio to a reasonable level. We have learned that the amplitude of the higher harmonics can be enhanced with specially micromachined cantilevers altered in such a way that the third flexural mode is an exact integer multiple of the fundamental resonance frequency. ${ }^{23}$ Simulations show that under these conditions the selected harmonic is very sensitive to material properties. ${ }^{24}$ Hereafter, we will designate the harmonics that match the frequency of a flexural mode as resonant harmonic. These special cantilevers enable a new imaging mode where we monitor the cantilever deflection at the harmonic corresponding to the third flexural mode. In this paper, we present a model for the response of a resonant harmonic in tapping-mode atomic force microscopy. We use this model to calculate the amplitude and phase of resonant harmonics for a variety of samples and demonstrate that the harmonics serve as a sensitive probe of material properties.

\section{THEORY}

In tapping mode, the cantilever is driven at the resonant frequency of the fundamental. When it is brought closer to the sample, the tip will periodically contact the sample. As the tip taps on the surface, the periodic impulse $\mathrm{s}^{25}$ will excite the flexural modes together with the higher harmonics. The amplitude of the harmonics is determined by a variety of parameters as outlined in a later section. Stark and Heckl ${ }^{26}$ 
have calculated the amplitudes of the higher harmonics by treating the tip-sample interaction as a linear spring and modeling the cantilever with a continuum mechanical system. In this paper we will follow the work of Sarid et al. ${ }^{27}$ and model the repulsive forces with the theory of Hertzian contacts and the attractive forces with the theory of Van der Waals interactions. We will use continuum mechanics to analyze a cantilever that have been modified so that the frequency of one of the higher flexural models matches the frequency of a harmonic of the fundamental frequency.

\section{A. Calculating the harmonics of tip-sample interaction forces}

Various aspects of the dynamics of tapping-mode atomic force microscopy (TM-AFM) have been studied in detail for real and model systems. ${ }^{28-35}$ Here we will calculate the time course of tip-sample interaction forces, and evaluate the harmonics with the Fourier transform.

We are able to use the harmonic approximation to calculate tip-sample interaction forces because the quality factor of the cantilevers is very high. Although tip-sample interaction contains several harmonics, it is mostly the fundamental harmonic at the driving frequency that affects the motion of the cantilever, because it is at the resonance frequency. Other harmonics act on the tip, but the frequency response of the cantilever at those frequencies is smaller (two to three orders of magnitude) than the response at the fundamental resonance frequency. Unfortunately, this approximation cannot be used for cantilevers immersed in liquids because the increased damping reduces the quality factor.

If we neglect the contribution of higher harmonics of the tip-sample interaction force on the cantilever motion, the problem is reduced to one of calculating the motion of a cantilever driven at its resonance frequency from both the base and tip. The driving force at the base will generate a free amplitude of $A_{0}$. The force at the tip is unknown and we are faced with the task of finding the magnitude and phase (relative to the phase of the cantilever motion) in terms of the free vibration amplitude, set-point amplitude $A_{s}$, phase of cantilever motion (relative to the driving signal), and the spring constant and quality factor of the cantilever. If we set the phase of the cantilever oscillation to zero, the motion of the cantilever can be written as $A_{s} e^{i \omega t}$. We represent the driving force and the fundamental harmonic of the tipsample force as $F_{d} e^{i(\omega t+\phi)}$ and $F_{\mathrm{ts} 1} e^{i(\omega t+\theta)}$, respectively,

$$
F_{T} e^{i(\omega t+\pi / 2)}=F_{d} e^{i(\omega t+\phi)}+F_{\mathrm{ts} 1} e^{i(\omega t+\theta)} .
$$

Here the $\pi / 2$ phase associated with the total force $F_{T}$ is due to the resonance of the cantilever, since on resonance the oscillations of the cantilever follow the total force with a phase delay of $\pi / 2$. Balantekin and Atalar ${ }^{36}$ used the phasor representation of Eq. (1) and studied the dynamics of a vibrating cantilever in noncontact. In Eq. (1), $F_{T}$ and $F_{d}$ can be written in terms of the spring constant $K_{1}$, quality factor $Q_{1}$, free amplitude $A_{0}$, and set-point amplitude $A_{s}$ as follows:

$$
\begin{aligned}
& F_{d}=K_{1} A_{0} / Q_{1}, \\
& F_{T}=K_{1} A_{s} / Q_{1} .
\end{aligned}
$$

Equation (2) describes a freely vibrating cantilever at resonance. In Eq. (3) we treat the cantilever as a linear system and the tip-sample force together with the driving force are the inputs to this system. So even though the tip-sample force is nonlinear, the total force and displacement of the cantilever satisfy the linear relation of Eq. (3). A similar approach is used by Stark et al. ${ }^{20}$ who modeled tip-sample forces as nonlinear feedback acting on the linear system of the cantilever. In Eq. (3), $F_{T}$ represents the sum of the nonlinear tip-sample forces and the driving force. Therefore, the output of the cantilever, which is the tip displacement, will satisfy the linear relation valid for a freely vibrating cantilever. In this approach, all the nonlinearity in the TM-AFM system is hidden in $F_{T}$. By writing Eq. (3), we are not neglecting the nonlinear contributions from the tip-sample interaction, however we are separating the linear and nonlinear parts of the mathematical problem to the solution.

When we substitute Eq. (2) and Eq. (3) into Eq. (1) and equate the real and imaginary parts of Eq. (1), we get the following relations:

$$
\begin{gathered}
\frac{K_{1} A_{0}}{Q_{1}} \cos \phi+F_{\mathrm{ts} 1} \cos \theta=0, \\
\frac{K_{1} A_{0}}{Q_{1}} \sin \phi+F_{\mathrm{ts} 1} \sin \theta=\frac{K_{1} A_{1}}{Q_{1}} .
\end{gathered}
$$

These equations relate the magnitude and phase of the fundamental harmonic of $f_{\text {ts }}$ to the known parameters of the tapping-mode operation. When we solve these equations for the magnitude $F_{\text {ts } 1}$ and phase $\theta$, we get

$$
\begin{gathered}
F_{\mathrm{ts} 1}=\frac{K_{1} A_{0}}{Q_{1}}\left[1-\frac{2 A_{s}}{A_{0}} \sin \phi+\frac{A_{s}^{2}}{A_{0}^{2}}\right]^{1 / 2}, \\
\theta=\tan ^{-1}\left(\frac{A_{s}-A_{0} \sin \phi}{A_{0} \cos \phi}\right) .
\end{gathered}
$$

These equations relate the magnitude and phase of the fundamental harmonic force to the measurable parameters of the cantilever.

An interesting and useful parameter in TM-AFM is the tip-sample energy dissipation due to the nonconservative nature of the interaction forces. We would like to calculate tip-sample energy dissipation with the sample approach as we did for the calculation of the fundamental harmonic of the tip-sample forces. Energy dissipation per oscillation cycle can be calculated by integrating the instantaneous power (the product of tip velocity and tip-sample force) over one cycle as

$$
E_{\mathrm{dis}}=-\int_{2 \pi / \omega} f_{\mathrm{ts}}(t) y(t) d t=\int_{2 \pi / \omega} A_{s} \omega f_{\mathrm{ts}} \sin (\omega t) d t .
$$

Here $y(t)$ is the first derivative of the position of the tip with respect to time. If $y(t)$ is chosen as $A_{s} \cos (\omega t)$, then $y(t)$ is equal to $-A_{s} \omega \cos (\omega t) . f_{\text {ts }}$ is the tip-sample force in time domain. Note that no particular interaction model is assumed and this equation is valid for any $f_{\text {ts }}$. The only approximation we make is the harmonic approximation, i.e., we assume 
a pure sinusoidal tip motion. Other than multiplicative terms, this integral is equivalent to the coefficient of $\sin (\omega t)$ in Fourier series expansion of $f_{\mathrm{ts}}$, which is equal to $-F_{\mathrm{ts} 1} \sin \theta$. Rewriting Eq. (8) with this replacement, we get

$$
E_{\mathrm{dis}}=-\pi A_{s} F_{\mathrm{ts} 1} \sin \theta .
$$

In this equation, $F_{\mathrm{ts} 1}$ is given by Eq. (6) and $\theta$ is given by Eq. (7). Note that for a dissipative $f_{\mathrm{ts}}, \sin (\theta)$ is always negative so that energy dissipation is a positive quantity. Inserting the values for $F_{\mathrm{ts} 1}$ and $\theta$ gives the more familiar relation for the energy dissipation per oscillation cycle for a cantilever driven at resonance, ${ }^{13,14}$

$$
E_{\mathrm{dis}}=\frac{\pi K_{1} A_{s}^{2}}{Q_{1}}\left[\frac{A_{0}}{A_{s}} \sin \phi-1\right] .
$$

This relationship has been derived earlier by considering the energy loss of tip-sample interaction. That we now find the same expression based on considerations of the tip-sample forces is an indication that our assumptions and model are correct. It is important to note the relation between phase $\phi$ of the cantilever relative to the driving force and phase $\theta$ of the tip-sample interaction forces relative to the cantilever motion. Equation (10) relates $\phi$ to the energy dissipation at the tip-sample contact. Because $A_{s}$ and $A_{0}$ are constants, we find in Eq. (7) that $\theta$ is also a measure of energy dissipation in the tip-sample contact. In fact, a nonzero $\theta$ means asymmetric tip-sample forces in approach and retraction of the tip, which in turn means that tip-sample forces are nonconservative.

Equation (6) shows that $F_{\mathrm{ts} 1}$ depends on the mechanical properties of the cantilever and the motion of the cantilever. Other than $\phi$, these parameters are independent of the surface properties. Since $\phi$ depends on the energy dissipation in tip-sample contact [see Eq. (10)], $F_{\text {ts1 }}$ also depends on tipsample energy dissipation.

In order to calculate the time course of interaction forces for a sample with known material properties from $F_{\text {ts }}$, we note that the time dependence of the tip-sample forces is determined by the maximum indentation depth (i.e., indentation of the sample when the tip is at its lowest position), which is equivalent to the minimum tip-sample separation in the attractive operation regime. There are two reasons for this. First, the cantilever motion remains nearly sinusoidal in typical tapping-mode operation when the cantilever has a high-quality factor, and second, the tip-sample force depends only on tip-sample separation and sample indentation. For a given depth of indentation, the time dependence of the tipsample separation and sample indentation is fully determined. With this information, an interaction model can be used to calculate the tip-sample forces. The interaction model we used for the tip-sample forces assumes a LennardJones type of distance dependence for the attractive forces, as shown below,

$$
f_{\mathrm{ts}}(r)=\frac{H R}{6 \sigma^{2}}\left[-\left(\frac{\sigma}{r}\right)^{2}+\frac{1}{30}\left(\frac{\sigma}{r}\right)^{8}\right] .
$$

Here $r$ is the tip-sample separation, $H$ is the Hamaker constant, $R$ is the tip radius, and $\sigma$ is the typical atomic distance for the tip and the surface. This force is attractive for tipsample separations larger than $r_{0}=30^{-1 / 6} \sigma$. To account for energy dissipation in tip-sample interaction, we used two $H$ values for the approach and retraction of the cantilever. This may not be the most realistic method for including the energy dissipation. However, energy dissipation plays an important role in the dynamics of tip motion as indicated by the hysteresis in adhesion forces that is observed in the tipsample interaction. For small separations, the sample is deformed under the influence of repulsive forces. The sample indentation with a Hertzian contact is approximately

$$
f_{\mathrm{ts}}(d)=\frac{4}{3} E \sqrt{R} d^{3 / 2},
$$

where $d$ is the deformation of the sample. The parameter $E$ is the reduced elastic modulus of the tip and is given by

$$
\frac{1}{E}=\frac{1-\nu_{t}^{2}}{E_{t}}+\frac{1-\nu_{s}^{2}}{E_{s}} .
$$

Here $E_{t}, \nu_{t}$ and $E_{s}, \nu_{s}$ are the elastic modulus and Poisson's ratios of the tip and the sample, respectively. We use Eq. (11) for tip-sample separations larger than $r_{0}$, where the force is attractive, and Eq. (12) for the positive repulsive force.

The tip-sample interaction model assumes that tip-sample energy dissipation is due to the attractive forces regardless of sample indentation. This simplifies the calculation, which is justified by noting that most of the samples energy is dissipated by capillary forces and hysteresis in the Van der Waals attractive force. With viscoelastic samples the energy is dissipated when the tip indents the sample. If we assume constant dissipation of the tip-sample energy, the phase of the cantilever motion $\phi$ is determined by Eq. (10). Equation (6) determines the interaction force $F_{\mathrm{ts} 1}$. Knowing $F_{\mathrm{ts} 1}$, we can calculate the tip-sample forces for increasing depths of indentation starting at 0 and increasing until the interaction force has a fundamental harmonic equal to the predetermined value of $F_{\mathrm{ts} 1}$. In the case of multiple solutions we pick the solution that belongs to the repulsive regime by looking at the sign of the average tip-sample force. The higher harmonic forces are calculated by taking the Fourier transform of the corresponding $f_{\text {ts }}$.

The tip-sample interactions for hard, medium, and soft samples are shown in Fig. 1. The Fourier components are calculated for a cantilever with spring constant $K_{1}=10$, quality factor $Q_{1}=100$, free amplitude $A_{0}=100 \mathrm{~nm}$, and setpoint amplitude $A_{s}=80 \mathrm{~nm}$. The reduced Young's modulus $E$ for the three samples is chosen to obtain contact durations of $5 \%, 10 \%$, and $15 \%$ of the period on the hard, medium, and soft samples, respectively. Attractive forces on all the samples are assumed to be equal for the same amount of energy dissipation at the contact. For the Hamaker constants we used $H_{a}=10 \times 10^{-20} \mathrm{~J}$ for approach and $H_{r}=30$ $\times 10^{-20} \mathrm{~J}$ for retraction. The parameters $R$ and $\sigma$ are chosen to be 10 and $0.1 \mathrm{~nm}$, respectively. These values result in an energy dissipation of approximately $30 \mathrm{eV}$ per tap.

According to Fig. 1, harmonics above the fifth are dependent on the hardness of the sample. Since the tip-sample 

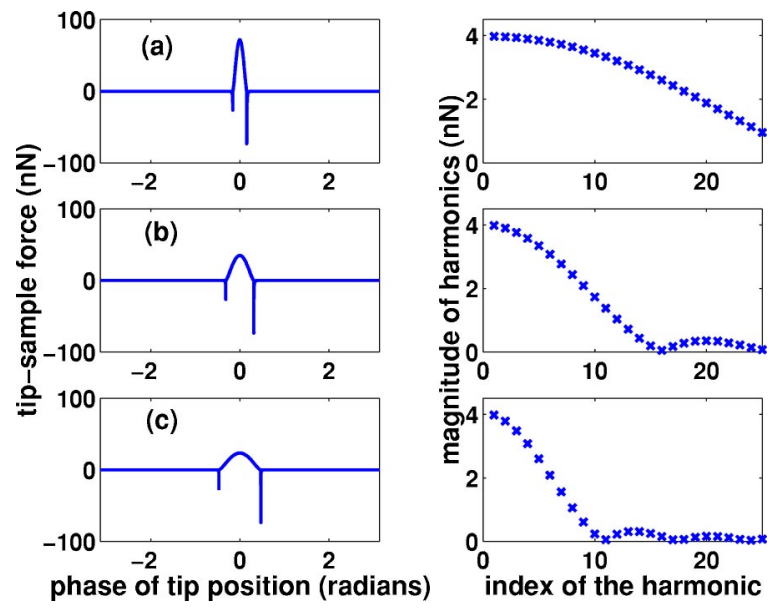

FIG. 1. (Color online) Interaction forces between the tip and the sample for three different samples: (a) hard, (b) medium, and (c) soft. The amplitude of the harmonics for the three tip-sample forces is shown on the right.

force is a periodic clipped sine wave, the width, or contact duration, determines the harmonic content; shorter pulses will generate larger amplitudes at higher harmonics. The duration of the contact increases for softer samples. We see in Fig. 1 that the first harmonic for each of the three cases has the same magnitude. This is because the magnitude of the first harmonic is given by Eq. (6) and an inspection of this equation shows that the only sample-dependent parameter is the phase $\phi$, which is a measure of energy dissipation. As previously mentioned, tip-sample dissipation is assumed to be constant for each of the three cases in Fig. 1. Therefore, the magnitudes of the first harmonics in each of the three cases are the same. These results show that the higher harmonics contain information on the sample stiffness.

\section{B. Mechanical model for the cantilever with higher-order modes}

In order to calculate the amplitude and phase of the cantilever response to the harmonic forces of the tip-sample interaction, we need to go beyond the simple harmonic oscillator and model the cantilever as a continuum mechanical system. The motion of the cantilever is governed by the Euler-Bernoulli equation. The solution of this equation for a rectangular cantilever can be found elsewhere. ${ }^{11,26,37}$ In Appendix A, we give a brief solution of the equation of motion for the cantilever when it is driven from its free end and we will use those results to describe the motion of the cantilevers that are in periodic contact with the sample.

The cantilever (Fig. 2) has several flexural modes of vibration. With the tip-sample interaction as the driving force, the motion of the cantilever can be expressed as a superposition of eigenmodes. With Eq. (A8) the response of the cantilever $y(x, t)$ to an external harmonic force applied to the point $x=L$ (tip) is expressed as

$$
y(x, t)=e^{i \omega t} \frac{F}{M} \sum_{n=1}^{\infty} \frac{Y_{n}(L) Y_{n}(x)}{\omega_{n}^{2}-\omega^{2}+i \omega / Q_{n}}(n=1,2,3, \ldots) .
$$

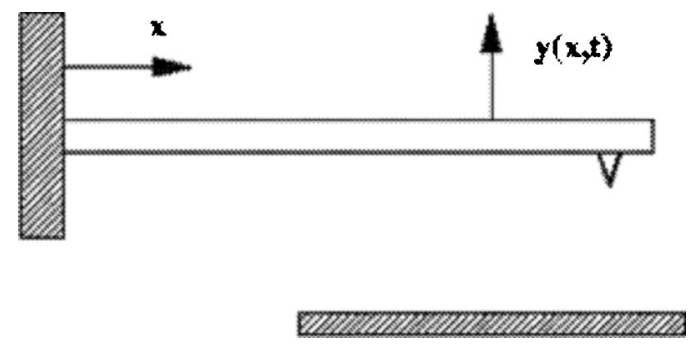

FIG. 2. Diagram of the cantilever-tip system tapping on a surface.

Here $y(x, t)$ is the displacement of the point $x$ on the cantilever at time t. $F$ and $\omega$ are the magnitude and frequency of the harmonic force. $Y_{n}, \omega_{n}$, and $Q_{n}$ are the mode shape, resonance frequency, and quality factor of the $n$th mode.

Under typical tapping-mode operating conditions, the quality factor of the resonance of the cantilever $Q_{n}$ is high (typically a few hundred). Therefore, a given mode will only be excited if the frequency of the harmonic force, $\omega$, is close to the resonance frequency, $\omega_{n}$, of that particular mode. Since we are interested in the tip displacement, we set $x$ $=L$. In addition, with Eq. (A3) we see that $Y_{n}^{2}(L)=4$ for all of the modes. Rewriting Eq. (14) with these changes, we get

$$
y(L, t)=e^{i \omega t} \frac{F}{M} \sum_{n=1}^{\infty} \frac{4}{\omega_{n}^{2}-\omega^{2}+i \omega / Q_{n}} .
$$

This result shows that the cantilever can be modeled by a series of harmonic oscillators each having an effective mass $m=M / 4$, resonance frequency $\omega_{n}$, and quality factor $Q_{n}$. The motion of the tip is a superposition of the displacement of many harmonic oscillators. In tapping mode there are several harmonic forces acting on the tip (see Fig. 1) and the actual displacement of the tip will be the sum of all the responses at the frequencies of the harmonics.

In the special case of the resonant harmonic, where $\omega_{n}$ is an integer multiple of the driving frequency, the response of the cantilever at that frequency will be dominated by the resonance of the $n$th flexural mode. Since one of the harmonics of the tip-sample interaction forces will match the resonance frequency $\omega_{n}$, the amplitude is enhanced by the quality factor of that mode. The other modes of the cantilever will not be excited by this harmonic force because they are off-resonance. Therefore, we can neglect the response of the other modes to the resonant harmonic in Eq. (15). The response of the cantilever at $\omega_{n}$ will be

$$
y(L, t)=e^{i \omega_{n} t} \frac{F_{n}}{M_{n}} \frac{Q_{n}}{i \omega_{n}^{2}} .
$$

Here $F_{n}$ is the amplitude of the resonant harmonic force, i.e., the Fourier component shown in Fig. 1 that matches the resonance frequency of the $n$th flexural mode. $M_{n}$ is the effective mass of the $n$th flexural mode, and is equal to $M / 4$ for a rectangular cantilever beam. The imaginary unit, $i$, appears since on resonance there is a $\pi / 2$ phase shift between the driving force and tip displacement. Rather than the mass and resonant frequency, we find it more convenient to work with 
the effective spring constants of the modes. Using the relation $\omega^{2}=k / m$ between mass, spring constant, and resonance frequency of a harmonic oscillator, we define an effective spring constant $K_{n}=M_{n} \omega_{n}^{2}$ for each flexural mode of the cantilever. The effective masses of the modes of a rectangular cantilever are the same and one can write

$$
\frac{K_{n}}{K_{1}}=\left(\frac{\omega_{n}}{\omega_{1}}\right)^{2}
$$

This equation relates the effective spring constant of a higher-order mode to the quantities $\omega_{1}, \omega_{n}$, and $K_{1}$. This equation provides a rough estimate but it is sufficient for our discussion of the potential of resonant harmonics for studying material properties.

\section{RESULTS AND DISCUSSION}

Now we have a complete formulation required to calculate the resonant harmonic response in tapping mode. We first calculate the tip-sample interaction forces and its harmonic content as described in the previous section and then we calculate the displacement of the cantilever at the resonant harmonic using Eq. (16). Using this methodology, we analyze the effects of the sample stiffness on the resonant harmonic. First, we calculate the resonant harmonic response as a function of reduced Young's modulus $E$. Based on the results of these calculations, we discuss how the amplitude and phase response relate to sample stiffness. Second, we study the effects of cantilever spring constant and set-point amplitude on the resonant harmonic response. Finally, we consider the harmonic number that is to be enhanced by a flexural resonance. We show that appropriate selection of cantilever spring constant and harmonic number can enhance the resolution of resonant harmonic AFM.

For a given Young's modulus we calculate the tip-sample interaction force $f_{\text {ts }}$ as described in the theory section. The harmonic forces are calculated by taking its Fourier transform. Because $f_{\text {ts }}$ is a periodic waveform, we can expand it into Fourier series as follows:

$$
f_{\text {ts }}(t)=\sum_{k=0}^{\infty} a_{n} \cos (k \omega t)+b_{n} \sin (k \omega t) \quad(k=0,1,2, \ldots),
$$

where the frequency $\omega$ is the driving frequency. Coefficients $a_{k}$ and $b_{k}$ are given as

$$
\begin{aligned}
& a_{k}=\frac{\omega}{\pi} \int_{0}^{2 \pi / \omega} f_{\mathrm{ts}} \cos (k \omega t) d t, \\
& b_{k}=\frac{\omega}{\pi} \int_{0}^{2 \pi / \omega} f_{\mathrm{ts}} \sin (k \omega t) d t .
\end{aligned}
$$

The $k$ th harmonic force can be written as

$$
F_{\mathrm{ts} k} \cos \left(k \omega t+\theta_{k}\right)=a_{k} \cos (k \omega t)+b_{k} \sin (k \omega t) .
$$

Here $F_{\mathrm{ts} k}=\sqrt{a_{k}^{2}+b_{k}^{2}}$ and $\theta_{k}$ are the magnitude and phase of the $k$ th harmonic. One of these harmonics will drive a
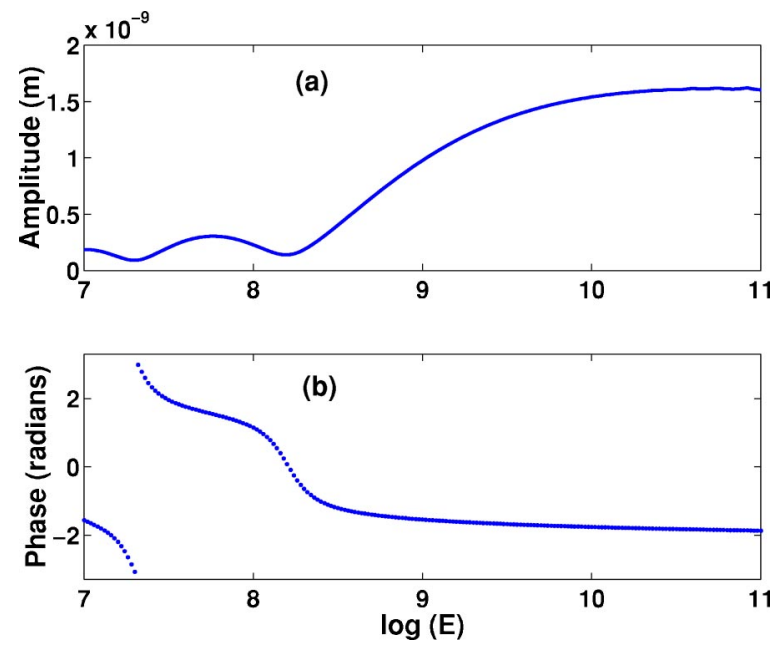

FIG. 3. (Color online) Amplitude (a) and phase (b) of the resonant harmonic frequency as a function of the stiffness. The unit of $E$ is Pascal and the base of the logarithm is 10 .

higher-order resonance that is specifically tuned to be an integer multiple of the fundamental resonance frequency of the cantilever (i.e., $k \omega=\omega_{n}, \omega_{n}$ is the resonance frequency of the $n$th flexural mode of the cantilever). Phase $\theta_{k}$ of a higher harmonic is defined relative to a reference signal at the same frequency as the higher harmonic. If we represent the tip displacement with $A_{s} \cos (\omega t)$, the reference signal will be $\cos (k \omega t)$. Note that for $k=1, F_{\text {ts } k}$ and $\theta_{k}$ are given by Eqs. (6) and (7).

In Fig. 3, we plot the amplitude and phase response of a resonant harmonic on samples with varying $E$ for a cantilever with a spring constant $K_{1}=1 \mathrm{~N} / \mathrm{m}$ and quality factor $Q_{1}=100$. The free amplitude and set-point amplitude are chosen to be $A_{0}=100 \mathrm{~nm}$ and $A_{s}=80 \mathrm{~nm}$, respectively. The Hamaker constants are $H_{a}=10 \times 10^{-20} \mathrm{~J}$ for the approach and $H_{r}=30 \times 10^{-20} \mathrm{~J}$ for the retraction. We use $\sigma=0.1 \mathrm{~nm}$ and $R=10 \mathrm{~nm}$ for the spacing and tip radius. These values correspond to an energy dissipation of approximately $30 \mathrm{eV}$ per tap. The resonant harmonic is 16 times the fundamental resonance frequency. This implies that the cantilever beam has been altered to tune the third flexural resonance frequency $\omega_{3}$ to 16 times the fundamental ${ }^{21}$ (i.e., $\omega_{3}=16 \omega$ ). The quality factor of the third-order resonance is assumed to be 600. According to Eq. (9), the effective spring constant of the third resonance is 256 times $K_{1}$. It is not necessary to specify a fundamental resonance frequency since Eq. (1) is independent of resonance frequency and since only the ratio of the frequencies appears in Eq. (17).

We see in Fig. 3 that the amplitude oscillates between minimum and maximum values with increasing values of $E$. In this range there are multiple values of $E$ that give the same amplitude, which complicates signal interpretation. For values of $E$ above the last minimum, the amplitude increases over a range of two orders of magnitude, and then saturates to a plateau. At the first minimum of Fig. 3, the contact time is approximately 2.5 times the period of the 16th harmonic. At the second minimum just before the plateau the contact time is 1.5 times the period of the 16 th harmonic. To under- 
stand the origin of the minima we consider Eqs. (19a) and (19b). If we assume that $f_{\mathrm{ts}}$ has a nonzero value only during contact, the intervals of the integrations can be reduced to the contact duration (i.e., pulse width of $f_{\text {ts }}$ ). If the period of the $k$ th harmonic is less than the contact duration, cosine and sine functions of Eq. (19) have both positive and negative contributions to the integration. At a certain contact duration, the positive and negative contributions will largely cancel, i.e., we get an amplitude minimum. For longer contact durations, the cosine and sine functions reverse signs multiple times, resulting in higher-order amplitude minima, as expected of Fourier transforms for periodic pulse waveforms. The important point here is that the contact time depends on the stiffness of the sample and the harmonic content is mainly determined by the contact time. This means that the harmonic amplitude is a function of the contact time. Since the contact time is related to the sample stiffness, we have gained a tool for monitoring the stiffness.

We now consider the origin of the phase shifts. In Eq. (19) we see that the coefficients $a_{k}$ and $b_{k}$ correspond to symmetric (even) and antisymmetric (odd) components of the tipsample interaction force $f_{\text {ts }}$ because $a_{k}$ uses the even function cosine and $b_{k}$ uses the odd function sine. If tip-sample interaction forces are equal in approach and retraction of the tip to the surface, $b_{k}$ and $\theta_{k}$ will be 0 . With energy dissipation in tip-sample forces, $b_{k}$ will be nonzero and $\theta_{k}$ will be a measure of the ratio of dissipative forces $\left(b_{k}\right)$ to conservative forces $\left(a_{k}\right)$. In addition to the phase of the resonant harmonic of $f_{\mathrm{ts}}$, there is an additional $\pi / 2$ phase delay in the response of the cantilever at the frequency of resonant harmonic because of the resonance of the cantilever at that frequency. In Fig. 3(b), we show the phase of the 16th harmonic. We see that the phase is changing with the stiffness of the surface even though the energy dissipation is constant at all values of $E$. The phase of 16th harmonic depends on the amount of energy dissipation as well as the time of dissipation. In our tip-sample interaction model, energy is dissipated just before the contact is broken (attractive forces are larger in retraction), and therefore the phase of the 16th harmonic contains information on the contact time. This produces a change in the variation of phase as $E$ changes. At each amplitude minima the phase change is faster with changes in $E$. Calculations for the case where there is no dissipation in the tip-sample interaction show $\pi$ phase shifts at the minima. These phase shifts are smoothed by finite dissipation.

In Fig. 4, we compare the amplitude and phase of the resonant harmonics for two different set-point amplitudes and two different cantilever spring constants. Figures 4(a) and 4(b) show the amplitude and phase responses for the varying set-point amplitude case $\left(A_{S}=60 \mathrm{~nm}\right.$ and $\left.80 \mathrm{~nm}\right)$. In Figs. 4(c) and 4(d) we show the amplitude and phase for two values of the spring constant case $\left(K_{1}=1\right.$ and $\left.10 \mathrm{~N} / \mathrm{m}\right)$. These figures show that with a stiffer cantilever and smaller set-point amplitude (with free amplitude held constant) the curves shift toward higher $E$. Both of these changes will increase the tip-sample force and, in turn, the contact time will increase, since the depth of the indent is increased. It follows that as the force increases, the sample stiffness must increase to maintain the same contact time.
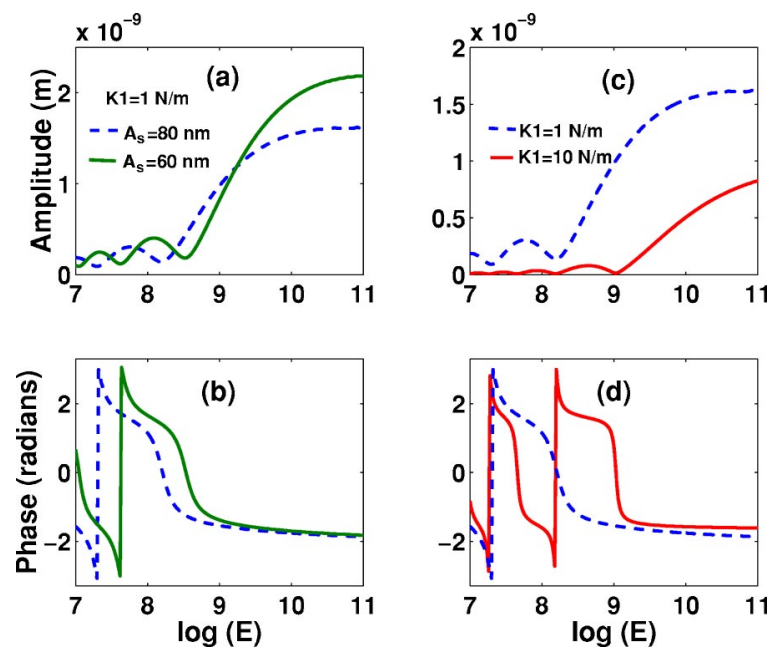

FIG. 4. (Color online) Amplitude and phase responses at the resonant harmonic for a cantilever at two different set-point amplitudes [(a) and (b)] and for two cantilevers with different spring constants [(c) and (d)]. The unit of $E$ is Pascal and the base of the logarithm is 10 .

For imaging we can record the amplitude or the phase of the resonant harmonic while scanning the surface in tapping mode. According to the results depicted in Figs. 3 and 4, the amplitude and phase signals are correlated with the stiffness of the surface in the vicinity of the tip. Therefore, an image generated by monitoring the resonant harmonic will map the elastic properties of the sample. One would prefer that the local stiffness values fall in the region between the last minimum and the plateau of Fig. 3. Preliminary knowledge of the nominal stiffness of the sample allows us to design a cantilever with the correct spring constant. In Fig. 4(c), we see that softer cantilevers will have the last minimum before the plateau at lower $E$ and stiff cantilevers have their last minimum at higher values of $E$. It is important to note that a cantilever that is too soft will reduce the sensitivity. For example, according to Fig. 4(c) a cantilever with $K_{1}=10 \mathrm{~N} / \mathrm{m}$ is most sensitive to stiffness variations around $10 \mathrm{GPa}$ while a cantilever with $K_{1}=1 \mathrm{~N} / \mathrm{m}$ is less sensitive in that range of materials. On the other hand, the cantilever with $K_{1}$ $=1 \mathrm{~N} / \mathrm{m}$ is sensitive to variations around $1 \mathrm{GPa}$. A proper value for the spring constant is crucial for operating in the monotonically increasing and highly sensitive region. This is not a very limiting constraint, because the monotonically increasing region extends over almost two orders of magnitude beyond the last amplitude minimum before it reaches the plateau (see Fig. 3). It is unlikely that the variations in a given sample will be this large. Although we need to use soft cantilevers for compliant samples and stiff cantilevers for hard samples, some flexibility is provided by adjusting the set-point amplitude to tune the sensitivity [see Fig. 4(a)].

Heretofore, the resonant harmonics were assumed to be at the 16th harmonic of the driving frequency. Now we would like to discuss the case where the frequency of the resonant harmonic is equal to other integer multiples of the fundamental resonance frequency. We have calculated the resonant harmonic response for cantilevers with their higher-order resonant frequencies at the 8th, 16th, and 24th harmonic. The 

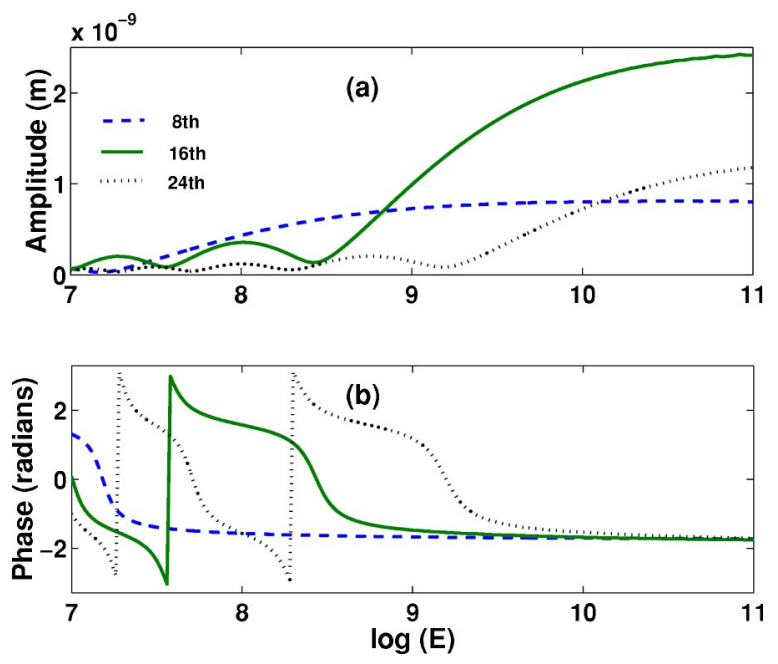

FIG. 5. (Color online) Amplitude (a) and phase (b) responses at the resonant harmonics when the resonant harmonics are located at the 8th, 16th, and 24th harmonics of the driving frequency. The amplitude response at the 8th harmonic is much higher than the others; therefore, we divided it by 10 in order to see all the responses clearly within one graph. The unit of $E$ is Pascal and the base of the logarithm is 10 .

cantilevers are assumed to all have the same spring constant for the fundamental vibration mode, $K_{1}=2 \mathrm{~N} / \mathrm{m}$. We are only interested in the general behavior of the resonantharmonic response at different integer multiples of the fundamental. The spring constant and quality factors of the higher-order resonances will affect the amplitude values, but they will not change the general trend of the amplitude and phase variations as the hardness of the surface changes. According to Eq. (17), the spring constant of the higher-order resonances of these cantilevers will be 128, 512, and 1152 $\mathrm{N} / \mathrm{m}$, respectively. For all three cantilevers, the set-point and free amplitudes are chosen as 80 and $100 \mathrm{~nm}$. The quality factors of the higher-order resonances are all assumed to be equal to 600. These assumptions for the spring constants and quality factors for the higher orders are not necessarily realistic but they simplify calculations. In Fig. 5 we summarize the results for three cases of the calculated resonant harmonic response. All three amplitude responses converge to their maximum as the hardness of the surface increases. As previously discussed in Fig. 3, the amplitude minimum occurs when contact duration and the period of the higher harmonic satisfy a certain ratio. Therefore, the 24th harmonic has its first amplitude minimum at a harder surface than the 16th and the 16th harmonic has its first minimum at a harder surface than the 8th harmonic. This result indicates that the 24th harmonic is more sensitive to harder samples and the 8 th harmonic is more sensitive to softer samples. This feature guides us in our choice of harmonics.

The amplitudes of the resonant harmonics saturate at a few nanometers, which is small compared to a set-point amplitude of $80 \mathrm{~nm}$. Since the depth of the indentation is comparable to these amplitudes, we expect that the time dependence of tip-sample interaction forces is affected by the highfrequency vibrations of the resonant higher-order modes. In a typical tapping-mode experiment, higher harmonics do not match the resonance frequencies of the higher-order modes. This results in relatively small amplitude at the higher harmonic and one can neglect the effect of high-frequency vibrations on the tip-sample forces. However, in the case of a resonant harmonic, the amplitude at that particular frequency is enhanced by the resonance of the cantilever. For a more detailed analysis one must incorporate the effects of the enhanced amplitude at the resonant harmonic on tip-sample forces.

It is important to note that there is a significant reduction in the noise floor for the frequencies near the resonant harmonic. Since the higher-order modes have effective spring constants much higher than the fundamental [see Eq. (9)], the vibration amplitude due to thermal noise is much smaller at those frequencies. There is a significant reduction in other sources of noise as well; the $1 / f$ noise is reduced since the signal has been moved to a higher frequency. Experimental results of Sahin et al. ${ }^{23}$ show that at the 16th harmonic, the noise floor is reduced by $30 \mathrm{~dB}$ as compared to the noise at the fundamental mode. This reduced noise floor means that even though the amplitudes at the resonant harmonics are relatively small, they offer an opportunity to measure the properties of the sample surface at the nanoscale.

\section{CONCLUSIONS}

We have presented a study of the cantilever motion in tapping-mode atomic force microscopy for a cantilever altered in such a way that the frequency of a harmonic of the fundamental mode matched the resonant frequency of a higher flexural mode. The results show that these resonant harmonics are sensitive to variations in the mechanical properties of materials. Since the amplitudes at the resonant harmonic are enhanced and the noise floor is reduced, there is a significant increase in the signal-to-noise ratio. The resonant harmonic response can be tuned for the desired application by selecting the correct value of the spring constant, the setpoint/free amplitude, and the higher harmonic. With this technique, elastic properties of very soft samples such as biological films and very hard samples such as semiconductor materials can be investigated with improved sensitivity.

\section{APPENDIX A}

Here we derive the equations governing the motion of a rectangular cantilever fixed at one end (base) and driven by an external force at the other end. The cantilever is a homogeneous rectangular elastic beam that has a width $a$, height $b$, and length $L$. The equation of motion for the flexural vibrations is given by the differential equation

$$
E I \frac{\partial^{4} y}{\partial x^{4}}+\gamma \frac{\partial y}{\partial t}+\rho A \frac{\partial^{2} y}{\partial t^{2}}=F \delta(x-L) e^{i \omega t}
$$

Here $E$ is the elasticity modulus, $\rho$ is the mass density, $I$ $=a b^{3} / 12$ is the area moment of inertia, and $A=a b$ is the cross section. $y(x, t)$ stands for the vertical displacement of the cantilever at position $x . F$ is the magnitude of the driving 
force and $\delta$ is the impulse function. Gamma represents the damping in the system. A better approximation for the damping results in a slightly more complicated equation of motion, the solution for which can be found in Ref. 26. Since the quality factors of cantilevers in air are relatively high, the effect of viscous damping and internal dissipation on the mode shapes and eigen-frequencies is negligible. Then the general solution to Eq. (A1) can be expressed as a superposition of the natural modes of the undamped cantilever as follows:

$$
y(x, t)=e^{-i \omega t} \sum_{n=1}^{\infty} P_{n} Y_{n}(x) .
$$

Here $Y_{n}(x)$ is the displacement of each natural mode and $P_{n}$ is an arbitrary coefficient that depends on the driving force. $Y_{n}(x)$ is given by

$$
\begin{aligned}
Y_{n}(x)= & \left(\frac{\sin \left(k_{n} L\right)-\sinh \left(k_{n} L\right)}{\cos \left(k_{n} L\right)+\cosh \left(k_{n} L\right)}\right)\left[\sin \left(k_{n} x\right)-\sinh \left(k_{n} x\right)\right] \\
& +\left[\cos \left(k_{n} x\right)-\cosh \left(k_{n} x\right)\right],
\end{aligned}
$$

where $k_{n}$ is the wave number satisfying the characteristic equation

$$
\cos \left(k_{n} L\right) \cosh \left(k_{n} L\right)+1=0 \quad\{n=1,2, \ldots\} .
$$

For each $k_{n}$ satisfying the above relation there is a corresponding natural mode of the cantilever and a resonance fre- quency $\omega_{n}$ that is determined by the dispersion relation $E I k^{4}-\rho A \omega^{2}=0$. By inserting Eq. (A3) into Eq. (A1) and eliminating the exponential time dependency, we get

$$
\sum_{n=1}^{\infty}\left(E I k_{n}^{4}-\rho A \omega^{2}\right) P_{n} Y_{n}(x)=F \delta(x-L) .
$$

In this equation the arbitrary coefficients $P_{n}$ can be found by using the orthogonality of the modes. That is, for any two modes, $Y_{m}(x)$ and $Y_{n}(x)$ will satisfy the condition

$$
\int_{0}^{L} Y_{m}(x) Y_{n}(x) d x=L \delta_{m n} .
$$

If we multiply both sides of Eq. (A5) with $Y_{m}(x)$ and integrate over the length of the cantilever, and using the relation given in Eq. (A6), we get

$$
P_{n}=\frac{F}{M} \frac{Y(L)}{\omega_{n}^{2}-\omega^{2}+i \omega \omega_{n} / Q_{n}} .
$$

The displacement of the cantilever $y(x, t)$ can be found by substituting $P_{n}$ and $Y_{n}$ into Eq. (A2). Then $y(x, t)$ will be given by

$$
y(x, t)=\frac{F e^{i \omega t}}{M} \sum_{n=1}^{\infty} \frac{Y(L) Y_{n}(x)}{\omega_{n}^{2}+i \omega \omega_{n} / Q_{n}-\omega^{2}} .
$$

*Corresponding author. Email address: sahin@stanford.edu

${ }^{1}$ G. Binnig, C. F. Quate, and Ch. Gerber, Phys. Rev. Lett. 56, 930 (1986).

${ }^{2}$ P. Maivald, H. J. Butt, S. A. C. Gould, C. B. Prater, B. Drake, J. A. Gurley, V. B. Elings, and P. K. Hansma, Nanotechnology 2, 103 (1993).

${ }^{3}$ M. Heuberger, G. Dietler, and L. Schlapbach, Nanotechnology 5, 12 (1994).

${ }^{4}$ A. J. Stephen and J. E. Houston, Rev. Sci. Instrum. 62, 710 (1990).

${ }^{5}$ H. U. Krotil, T. Stifter, H. Waschipky, K. Weishaupt, S. Hild, and O. Marti, Surf. Interface Anal. 27, 336 (1999).

${ }^{6}$ K. Yamanaka, H. Ogiso, and O. Kosolov, Appl. Phys. Lett. 64, 178 (1994).

${ }^{7}$ K. Yamanaka and S. Nakano, Jpn. J. Appl. Phys., Part 1 35, 3787 (1996)

${ }^{8}$ O. V. Kolosov, M. R. Castell, C. D. Marsh, G. A. D. Briggs, T. I. Kamins, and R. S. Williams, Phys. Rev. Lett. 81, 1046 (1998).

${ }^{9}$ K. B. Crozier, G. G. Yaralioglu, F. L. Degertekin, J. D. Adams, S. C. Minne, and C. F. Quate, Appl. Phys. Lett. 76, 1950 (2000).

${ }^{10}$ G. G. Yaralioglu, F. L. Degertekin, K. B. Crozier, and C. F. Quate, J. Appl. Phys. 87, 7491 (2000).

${ }^{11}$ U. Rabe, K. Janser, and W. Arnold, Rev. Sci. Instrum. 67, 3281 (1996)

${ }^{12}$ Q. Zhong, D. Inniss, K. Kjoller, and V. B. Elings, Surf. Sci. 280, L688 (1993).

${ }^{13}$ J. P. Cleveland, B. Anczykowski, A. E. Schmid, and V. B. Elings,
Appl. Phys. Lett. 72, 2613 (1998).

${ }^{14}$ J. Tamayo and R. Garcia, Appl. Phys. Lett. 73, 2926 (1998).

${ }^{15}$ L. Zitzler, S. Herminghaus, and F. Mugele, Phys. Rev. B 66, 155436 (2002).

${ }^{16}$ M. Balantekin and A. Atalar, Phys. Rev. B 67, 193404 (2003).

${ }^{17}$ M. Stark, C. Moeller, D. J. Mueller, and R. Guckenberger, Biophys. J. 80, 3009 (2001).

${ }^{18}$ T. R. Rodriguez and R. Garcia, Appl. Phys. Lett. 84, 449 (2004).

${ }^{19}$ R. Hillenbrand, M. Stark, and R. Guckenberger, Appl. Phys. Lett. 76, 3478 (2000).

${ }^{20}$ M. Stark, R. W. Stark, W. M. Heckl, and R. Guckenberger, Proc. Natl. Acad. Sci. U.S.A. 99, 8473 (2002).

${ }^{21}$ R. W. Stark and W. M. Heckl, Rev. Sci. Instrum. 74, 5111 (2003).

${ }^{22}$ T. Rodriguez and R. Garcia, Appl. Phys. Lett. 80, 1646 (2002).

${ }^{23}$ O. Sahin, G. Yaralioglu, R. Grow, S. F. Zappe, A. Atalar, C. F. Quate, and O. Solgaard, TRANSDUCERS '03 (IEEE, Boston, 2003), p. 1124.

${ }^{24}$ O. Sahin and A. Atalar, Appl. Phys. Lett. 79, 4455 (2001).

${ }^{25}$ M. V. Salapaka, D. J. Chen, and J. P. Cleveland, Phys. Rev. B 61, 1106 (2000).

${ }^{26}$ R. W. Stark and W. M. Heckl, Surf. Sci. 457, 219 (2000).

${ }^{27}$ D. Sarid, T. G. Rushell, R. K. Workman, and D. Chen, J. Vac. Sci. Technol. B 14, 864 (1996).

${ }^{28}$ A. Kuhle, A. H. Sorensen, and J. Bohr, J. Appl. Phys. 81, 6562 (1997).

${ }^{29}$ R. Garcia and A. SanPaulo, Phys. Rev. B 60, 4961 (1999).

${ }^{30}$ O. Sahin and A. Atalar, Appl. Phys. Lett. 78, 2973 (2001). 
${ }^{31}$ J. Tamayo and R. Garcia, Langmuir 12, 4430 (1996).

${ }^{32}$ A. SanPaulo and R. Garcia, Phys. Rev. B 64, 193411 (2001).

${ }^{33}$ A. SanPaulo and R. Garcia, Phys. Rev. B 66, 041406(R) (2002).

${ }^{34}$ M. Marth, D. Maier, J. Honerkamp, R. Brandach, and G. Bar, J. Appl. Phys. 85, 7030 (1999).
${ }^{35}$ L. Wang, Appl. Phys. Lett. 73, 3781 (1998).

${ }^{36}$ M. Balantekin and A. Atalar, Appl. Surf. Sci. 205, 86 (2003).

${ }^{37}$ J. A. Turner, S. Hirsekorn, U. Rabe, and W. Arnold, J. Appl. Phys. 82, 966 (1997). 